\title{
Quantum phase transitions in two-dimensional tilted optical lattices
}

\author{
Andrey R. Kolovsky \\ Kirensky Institute of Physics, 660036 Krasnoyarsk, Russia \\ and Siberian Federal University, 660041 Krasnoyarsk, Russia
}

(Received 21 October 2015; published 14 March 2016)

\begin{abstract}
We discuss the quantum phase transition between the Mott-insulator state and the density-wave state of cold Bose atoms in a two-dimensional (2D) square lattice as the lattice is adiabatically tilted along one of its primary axes. It is shown that a small misalignment of the tilt drastically changes the result of the adiabatic passage and, instead of the density-wave state, one obtains a disordered state. An intrinsic relation of the problem to Bloch oscillations of hard-core bosons in a 2D lattice is illuminated.
\end{abstract}

DOI: 10.1103/PhysRevA.93.033626

\section{INTRODUCTION}

Tilted one-dimensional (1D) optical lattices are the standard setup for studying Bloch oscillations (BOs) of noninteracting and interacting cold atoms [1-7], with important applications to precision measurements of the gravitational force [6] and interatomic interaction constant [7]. A different direction of research is quantum phase transitions in tilted lattices [8,9]. (We also mention relevant studies of the quench dynamics [10-15].) These are rather specific phase transitions because, formally, the system has no ground state. Nevertheless, by adiabatically tilting the lattice one observes continuous evolution of the Mott-insulator (MI) state of Bose atoms into the density-wave (DW) state [9,14]. As it was explained in Ref. [8], by mapping the system of Bose atoms into an effective system of interacting spins, this "not-ground-state" transition corresponds to the common ground-state phase transition from ferromagnetic to antiferromagnetic ordering of the Heisenberg spin chain.

More phases are expected if we consider Bose atoms in two-dimensional (2D) lattices [16]. In fact, two-dimensional systems offer a freedom in choosing the lattice geometry and orientation of a static force $\mathbf{F}$ relative to primary axes of the lattice. However, since we face not-ground-state transition, this freedom may lead to additional effects that are absent in the effective ground-state problem. In this work we discuss one of them, namely, an effect caused by the lattice misalignment. In more detail, we shall analyze formation of the density-wave phase in a square lattice which is tilted in the $y$ direction with some uncertainty $F_{x} \ll F_{y}$ (see Fig. 1). It will be shown that this small misalignment completely changes the result of the adiabatic passage and, instead of the ordered DW state, one obtains a disordered state.

\section{PHASE TRANSITION IN A LADDER}

To illustrate the role of the weak component $F_{x}$ we first consider the square lattice which consists of two rows, i.e., a two-leg ladder. Using the creation, $\hat{a}_{l, m}^{\dagger}$, and annihilation, $\hat{a}_{l, m}$, bosonic operators and denoting by $\hat{n}_{l, m}$ the number operator, $\hat{n}_{l, m}=\hat{a}_{l, m}^{\dagger} \hat{a}_{l, m}$, the Hamiltonian of interacting Bose atoms in the tilted ladder can be written in the form

$$
\begin{aligned}
\widehat{H}(t)= & -\frac{J_{x}}{2} \sum_{m=1}^{2} \sum_{l=1}^{L}\left(\hat{a}_{l+1, m}^{\dagger} \hat{a}_{l, m} e^{-i \frac{F_{x} d}{\hbar} t}+\text { H.c. }\right) \\
& -\frac{J_{y}}{2} \sum_{l=1}^{L}\left(\hat{a}_{l, 2}^{\dagger} \hat{a}_{l, 1}+\text { H.c. }\right)+\frac{U}{2} \sum_{l, m} \hat{n}_{l, m}\left(\hat{n}_{l, m}-1\right) \\
& -F_{y} d \sum_{l}\left(\hat{n}_{l, 2}-\hat{n}_{l, 1}\right) .
\end{aligned}
$$

In the Hamiltonian (1) the first term describes the hopping of atoms along the ladder legs (the $x$ direction) with the rate $\hbar / J_{x}$, the second term is the hopping between the ladder legs (the $y$ direction) with the rate $\hbar / J_{y}$, the third one is the on-site interaction energy with $U$ being the microscopic interaction constant, the fourth term is the energy mismatch between the legs due to the tilt in the $y$ direction, and the weak component $F_{x}$ is taken into account in the first term by using the gauge transformation. (In what follows we set the lattice period $d$ and the Planck constant $\hbar$ to unity.) In numerical simulations we use the periodic boundary conditions in the $x$ direction, which are known to facilitate convergence of results in the thermodynamic limit $L \rightarrow \infty$. We mention that the system (1) can be realized experimentally by using a double-periodic optical potential in the $y$ direction which splits the 2D lattice into an array of independent two-leg ladders [17].

Since we are interested in the case $U \gg J_{x}, J_{y}$, we can truncate the Hilbert space to the resonant subspace spanned by the Fock states

$$
|\mathbf{n}\rangle=\left[\begin{array}{llll}
n_{1,2} & n_{2,2} & \ldots & n_{L, 2} \\
n_{1,1} & n_{2,1} & \ldots & n_{L, 1}
\end{array}\right]
$$

where occupation numbers $n_{l, m}$ may take value one or zero if $m=1$ and one or two if $m=2$. Then the discussed phase transition corresponds to evolution of the MI state, where $n_{l, m}=1$, into the ordered doublon state, where $n_{l, 1}=0$ and $n_{l, 2}=2$. This evolution is illustrated in Fig. 2 where $F_{x}=0$ and we increase $F_{y}$ linearly in time with the rate $v=0.0005$. Different curves in the lower panel in Fig. 2 are probabilities 


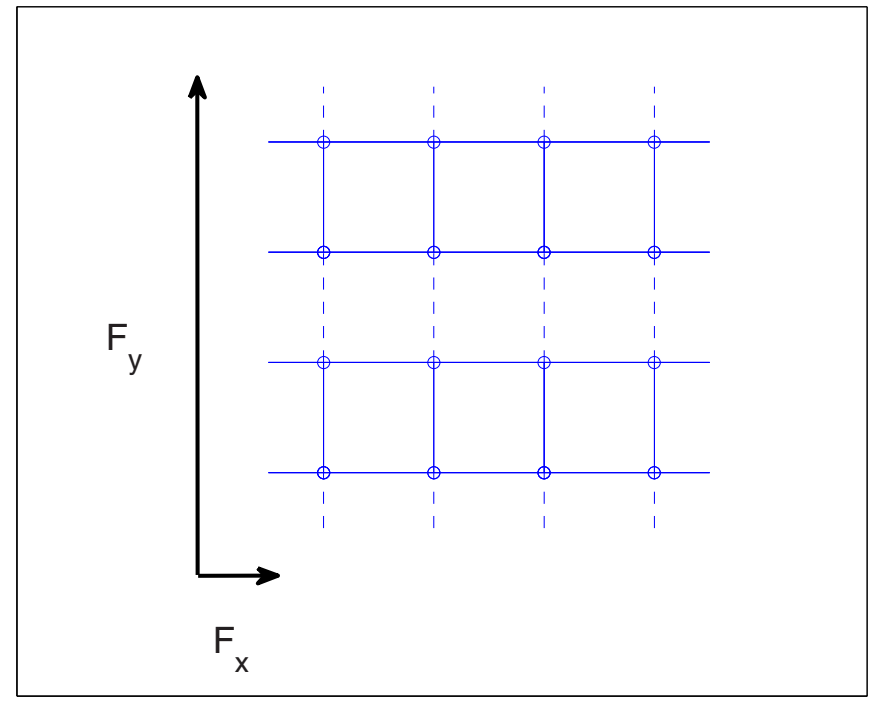

FIG. 1. A sketch of the considered system. Strongly interacting Bose atoms in a square optical lattice are subject to a static force $F$ which is slightly misaligned with the primary $y$ axis. A simpler problem corresponds to an array of independent two-leg ladders which are obtained from the square lattice by setting the hopping matrix elements for the dashed-line bonds to zero.

$P_{n}(t)$ to find $n$ doublons in the ladder at a given time. The solid line in the upper panel is the total number of doublons normalized to the lattice size:

$$
N_{d}(t)=\frac{1}{L} \sum_{n=0}^{L} n P_{n}(t)
$$
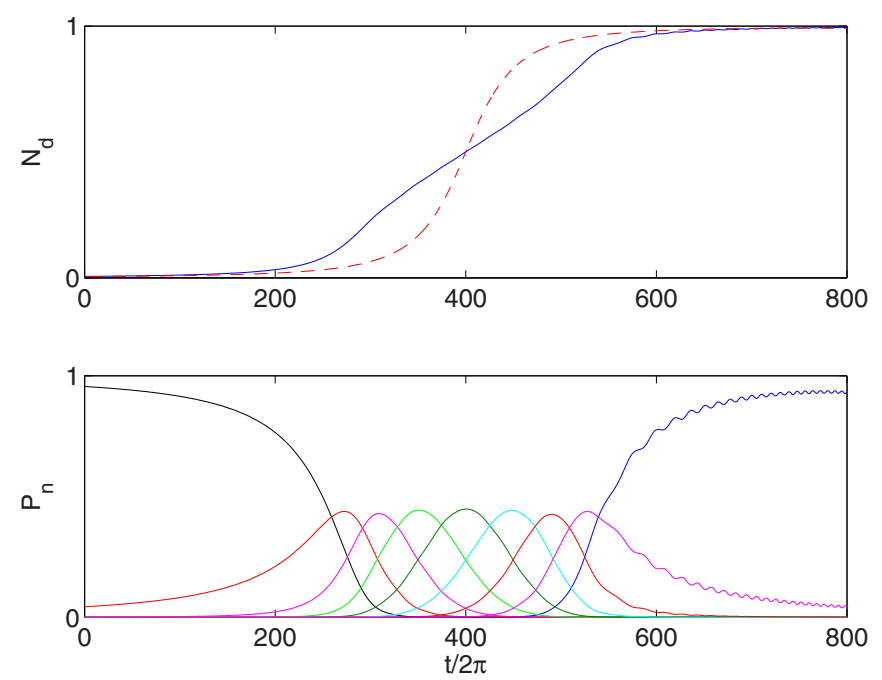

FIG. 2. Probabilities $P_{n}(t)$ to find $n$ doublons in the system, lower panel, and total number of doublons Eq. (3), upper panel. The field component $F_{y}$ is increased linearly in time, where the depicted time interval corresponds to $0.8 \leqslant F_{y} \leqslant 1.2$. The energy and time units are given by the value of the interaction constant $U$ which we set to unity. The other parameters are $F_{x}=0, L=8$, and $J_{x}=J_{y}=0.02$. The dashed line in the upper panel corresponds to the case $J_{x}=0$.
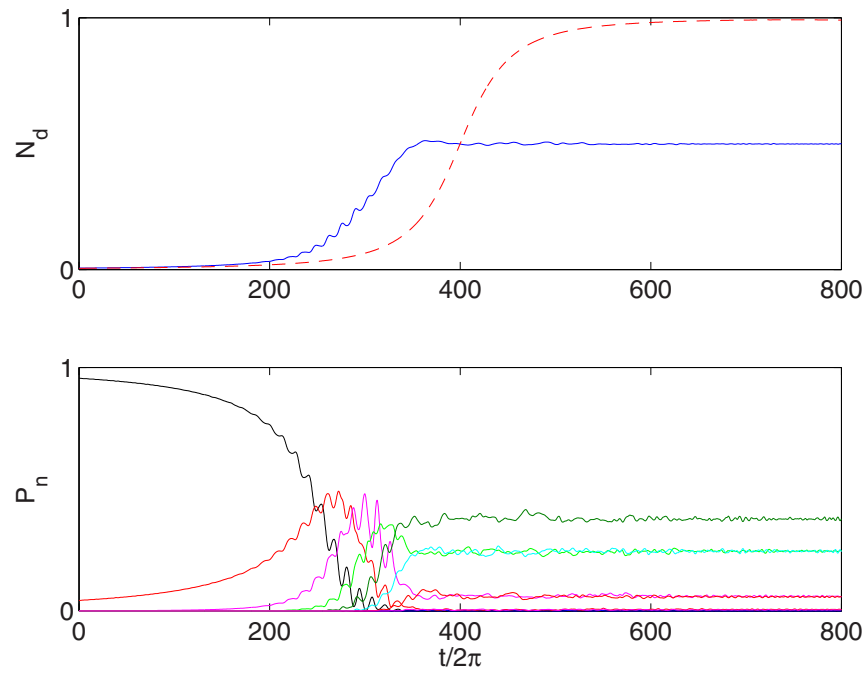

FIG. 3. The same as in Fig. 2 but $F_{x}=0.01$.

It is seen in Fig. 2 that we obtain the DW state at the end of the adiabatic passage. We note that if we set $J_{x}$ to zero the problem will reduce to two bosons in an asymmetric double-well potential. Qualitatively this toy system reproduces the discussed phase transition (see the dashed line in the upper panel), where the main difference is that for $J_{x} \neq 0$ the transition region is wider. Let us also mention that the result for $N_{d}(t)$ depicted in the upper panel is converged in the thermodynamic sense, i.e., the further increase of the ladder size $L$ does not affect the shape of the curves.

Dynamics of the system (1) changes drastically if $F_{x} \neq 0$ (see Fig. 3). Now the final state is a random state from the microcanonical distribution, where probabilities $P_{n}$ are given by relative dimensions of the corresponding subspaces of the Hilbert space:

$$
P_{n}=\frac{\mathcal{N}_{n}}{\mathcal{N}}, \quad \mathcal{N}=\sum_{n=0}^{L} \mathcal{N}_{n}, \quad \mathcal{N}_{n} \approx \frac{1}{L}\left(\frac{L !}{n !(L-n) !}\right)^{2}
$$

(The prefactor $1 / L$ in the last equation is due to periodic boundary conditions which restrict the system dynamics to zero-quasi-momentum subspace.) For $L=8$ the total dimension of the zero-quasi-momentum Hilbert space $\mathcal{N}=$ 1620 and $\mathcal{N}_{n}=1,8,100,392,618,392,100,8,1$, respectively. This gives $P_{4} \approx 0.38, P_{3}=P_{5} \approx 0.24, P_{2}=P_{6} \approx 0.06, P_{1}=$ $P_{7} \approx 0.005$, and $P_{0}=P_{8} \approx 0$, which are in perfect agreement with the numerical result shown in the lower panel in Fig. 3. As it will be explained below, the physics behind this result is self-thermalization of the system due to BOs of the quasiparticles (doublons and holes) in the $x$ direction. These quasiparticles are dynamically created when we tilt the ladder in the $y$ direction. Since quasiparticles behave as hard-core (HC) bosons, one gets useful insight into the problem by studying BOs of $\mathrm{HC}$ bosons in a ladder. 


\section{BLOCH OSCILLATIONS OF HC BOSONS}

Bloch dynamics of $\mathrm{HC}$ bosons in a ladder is governed by the time-dependent Hamiltonian

$$
\begin{aligned}
\widehat{H}(t)= & -\frac{J_{x}}{2} \sum_{m=1}^{2} \sum_{l=1}^{L}\left(\hat{b}_{l+1, m}^{\dagger} \hat{b}_{l, m} e^{-i F t}+\text { H.c. }\right) \\
& -\frac{J_{y}}{2} \sum_{l=1}^{L}\left(\hat{b}_{l, 2}^{\dagger} \hat{b}_{l, 1}+\text { H.c. }\right)
\end{aligned}
$$

where $\hat{b}_{l, m}^{\dagger}$ and $\hat{b}_{l, m}$ are the hard-core creation and annihilation bosonic operators, $\left(\hat{b}_{l, m}^{\dagger}\right)^{2}=0$, and $F \equiv F_{x}$ is a static field parallel to the ladder legs. Referring to the original system (1) the model (2) mimics the case of fixed $F_{y}=U$ where the double-well potential becomes symmetric. We shall restrict ourselves by the filling factor $1 / 2$ because in this case the Hilbert space of the system (5) and the system (1) are isometric. In fact, let

$$
|\mathbf{n}\rangle=\left[\begin{array}{llll}
n_{1,2} & n_{2,2} & \ldots & n_{L, 2} \\
n_{1,1} & n_{2,1} & \ldots & n_{L, 1}
\end{array}\right], \quad n_{l, m}=0,1
$$

be the complete set of Fock states of HC bosons at half filling. Then the resonant subset of Fock states of the system (1) is obtained by adding unity to every element in the upper row, leaving the lower row unchanged. The isometric Hilbert spaces imply the same skeleton of the Hamiltonian matrix, although the values of nonzero matrix elements may differ by factor $\sqrt{2}$ due to bosonic enhancement of tunneling in the original problem.

It is well known that $\mathrm{HC}$ bosons in a 1D lattice can be mapped into the system of noninteracting fermions. This is, however, not the case for HC bosons in a ladder. Here any attempt of mapping leads to effective interactions and, thus, we are faced with BOs of interacting particles. Previous studies of Bloch dynamics of interacting atoms revealed two qualitatively different regimes of BOs [3-5,7]. These are the quasiperiodic BOs, which take place for a strong field $F$, and decaying BOs, which is the case for a weak field. In the latter case the system gets thermalized, i.e., every Fock state becomes equally occupied [5]. We found remarkable similarities between BOs of $\mathrm{HC}$ bosons in a ladder and BOs of weakly $(U \sim J)$ interacting bosons in a $1 \mathrm{D}$ lattice. In particular, depending on the field strength, BOs of HC bosons in the ladder either irreversibly decay or show a quasiperiodic dynamics (see Fig. 4). In the former case the irreversible decay indicates self-thermalization of the system. Using the above-mentioned similarity with the original problem this explains the result (4).

\section{INFINITE 2D LATTICES}

The above analysis of the ladder system suggests the following picture of phase transition in the 2D lattice. When we tilt the lattice in the $y$ direction to $F_{y} \approx U$ we produce particle-hole excitations of the MI state. These quasiparticles can move in the $x$ direction and, if $F_{x} \neq 0$, this motion causes self-thermalization of the system within the characteristic time $T_{B}=2 \pi / F_{x}$. To avoid this self-thermalization, the evolution time must be smaller than the Bloch period $T_{B}$. On the other hand, to ensure adiabatic passage between the MI and
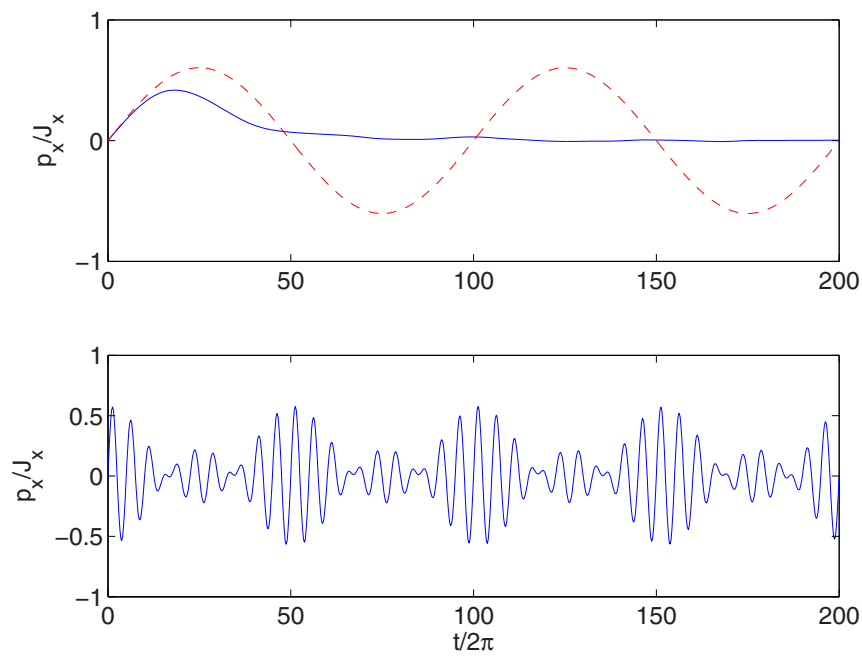

FIG. 4. Bloch oscillations of HC bosons in a two-leg ladder at half filling. The mean momentum per particle is shown as a function of time. Parameters are $J_{x}=J_{y}=0.02, L=N=8, F=0.01$, upper panel, and $F=0.2$, lower panel. The initial wave function is given by the ground state of the system for $F=0$. The dashed line in the upper panel denotes BOs of spinless fermions at half filling.

DW states, this time should be as large as possible. These contradicting conditions introduce severe restriction on the lattice misalignment.

We mimic phase transition in an infinite lattice by considering finite lattices up to $4 \times 4$ sites and imposing periodic boundary conditions in both the $x$ and $y$ directions. Then the static force $F=v t$ appears as oscillating phases $\exp \left(-i \beta v t^{2} / 2\right)$ and $\exp \left(-i \sqrt{1-\beta^{2}} v t^{2} / 2\right)$ (here $\left.\beta=F_{x} / F_{y}\right)$ in the hopping terms of the Bose-Hubbard Hamiltonian and we used the Runge-Kutta method of the fourth order to solve the resulting nonstationary Schrödinger equation. We also truncate the Hilbert space to the doublon subspace [19]. To characterize the final state of the system we introduce the order parameters

$$
\begin{aligned}
& D_{x}(t)=N^{-1}\left\langle\psi(t)\left|\sum_{l, m} \hat{n}_{l+1, m} \hat{n}_{l, m}\right| \psi(t)\right\rangle, \\
& D_{y}(t)=N^{-1}\left\langle\psi(t)\left|\sum_{l, m} \hat{n}_{l, m+1} \hat{n}_{l, m}\right| \psi(t)\right\rangle,
\end{aligned}
$$

where $N$ is the number of atoms coinciding with the number of sites. It is easy to prove that the MI state corresponds to $D_{x}=D_{y}=1$ while the DW state has $D_{x}=2$ and $D_{y}=0$. For $F_{x}=0$ (i.e., the precise alignment) and the rate $v=0.001$ dynamics of the order parameters (7) and (8) is depicted in the upper panel in Fig. 5. First of all we notice that the final state deviates from the ideal DW state even for $F_{x}=0$. This is a consequence of the high-order resonant tunneling which happens at $F=U / j$ where $j$ is an integer number (see the recent works $[14,15]$ and references therein). For the considered rate $v=10^{-3}$ only the second-order process is important. It creates a small number of doublons and holes in the next-nearest rows of the lattice as $F$ is increased above $U / 2$. For the subsequent first-order process at $F=U$ these objects play the role of defects which prohibit particle-hole excitations of the MI state in their vicinity. For this reason 

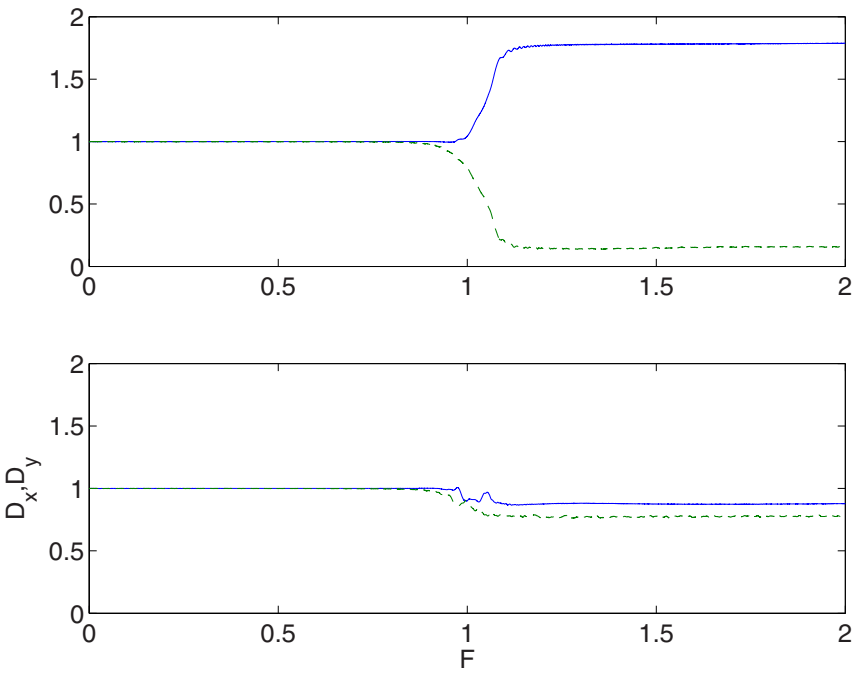

FIG. 5. Order parameters $D_{x}(t)$, solid lines, and $D_{y}(t)$, dashed lines, for $F_{x} / F_{y}=0$, upper panel, and $F_{x} / F_{y}=0.01 / \sqrt{1-0.01^{2}}$, lower panel. The static field is increased linearly in time with the rate $v=10^{-3}$, and the lattice size is $3 \times 4$.

the total number of doublons never reaches the maximally possible number $N / 2$ and the order parameters deviate from their extreme values.

Small deviation of the final state from the DW state due to the high-order resonant tunneling is a minor effect in comparison with the effect of the lattice alignment. The lower panel in Fig. 5 shows the result of numerical simulations for $\beta=F_{x} / F_{y} \approx 0.01$. It is seen that the final state practically has no correlations. At the same time, the total number of doublons remains pretty high (results are not shown). Thus we end up with a disordered state of doublons.

\section{CONCLUSION}

We analyzed response of the Mott-insulator state of cold atoms in a square 2D optical lattice to a static field $\mathbf{F}$ which is adiabatically increased from zero to a value above the interaction energy $U$. If the field $\mathbf{F}$ is precisely aligned with the $y$ axis of the lattice the Mott-insulator state was shown to evolve in the density-wave state where every second row is empty and the remaining rows are filled with doublons (two atoms in one site). This result can be viewed as twodimensional generalization of the quantum phase transition observed in tilted 1D optical lattices $[9,14]$. The new effect was found if the static field is slightly misaligned with respect to the $y$ axis. In this case the final state of the system is a disordered state of doublons and holes with vanishing correlations. The physics behind this effect proved to be self-thermalization of the system due to Bloch oscillations of the quasiparticles (doublons and holes) in the $x$ direction.

In the present work we restricted ourselves by considering the field orientation close to the primary $y$ axis of the square 2D lattice. Obviously, all reported results hold true in the situation where $\mathbf{F}$ is close the $x$ axis. The case of other orientations, for example, $F_{x} / F_{y} \approx 1$, is more involved and is expected to strongly depend on the lattice geometry. In fact, the simple square lattice is a rare exclusion where single-particle wave functions, known as the Wannier-Stark states, are localized for any orientations of the static field except those coinciding with primary axes. In a generic 2D lattice the quantum particle is delocalized in the direction orthogonal $\mathbf{F}$ for every "rational" orientation of the static field which is given by arbitrary superposition of the translation vectors [18]. Thus one may expect a similar result: we shall observe transition to an ordered state if the vector $\mathbf{F}$ points from one lattice site to a nearby lattice site exactly and self-thermalization of the system if $\mathbf{F}$ slightly deviates from this direction. The detailed analysis of the outlined problem will be presented elsewhere.

\section{ACKNOWLEDGMENTS}

The author acknowledges fruitful discussions with M. Fleischhauer and F. Grusdt; the hospitality of the University of Kaiserslautern, where a part of this work was conducted; and financial support from Russian Foundation for Basic Research through Project No. 15-02-00463, Wannier-Stark states and Bloch oscillations of a quantum particle in a generic two-dimensional lattice.
[1] M. Ben Dahan, E. Peik, J. Reichel, Y. Castin, and C. Salomon, Bloch Oscillations of Atoms in an Optical Potential, Phys. Rev. Lett. 76, 4508 (1996).

[2] O. Morsch, J. H. Müller, M. Cristiani, D. Ciampini, and E. Arimondo, Bloch Oscillations and Mean-Field Effects of BoseEinstein Condensates in 1D Optical Lattices, Phys. Rev. Lett. 87, 140402 (2001).

[3] A. R. Kolovsky, New Bloch Period for Interacting Cold Atoms in 1D Optical Lattices, Phys. Rev. Lett. 90, 213002 (2003)

[4] A. Buchleitner and A. R. Kolovsky, Interaction-Induced Decoherence of Atomic Bloch Oscillations, Phys. Rev. Lett. 91, 253002 (2003).

[5] A. R. Kolovsky, H. J. Korsch, and E.-M. Graefe, Bloch oscillations of Bose-Einstein condensates: Quantum counterpart of dynamical instability, Phys. Rev. A 80, 023617 (2009).
[6] N. Poli, F.-Y. Wang, M. G. Tarallo, A. Alberti, M. Prevedelli, and G. M. Tino, Precision Measurement of Gravity with Cold Atoms in an Optical Lattice and Comparison with a Classical Gravimeter, Phys. Rev. Lett. 106, 038501 (2011).

[7] F. Meinert, M. J. Mark, E. Kirilov, K. Lauber, P. Weinmann, M. Gröbner, and H.-C. Nägerl, Interaction-Induced Quantum Phase Revivals and Evidence for the Transition to the Quantum Chaotic Regime in 1D Atomic Bloch Oscillations, Phys. Rev. Lett. 112, 193003 (2014).

[8] S. Sachdev, K. Sengupta, and S. M. Girvin, Mott insulators in strong electric fields, Phys. Rev. B 66, 075128 (2002).

[9] J. Simon, W. S. Bakr, R. Ma, M. E. Tai, P. M. Preiss, and M. Greiner, Quantum simulation of antiferromagnetic spin chains in an optical lattices, Nature (London) 472, 307 (2011). 
[10] K. Sengupta, S. Powell, and S. Sachdev, Quench dynamics across quantum critical points, Phys. Rev. A 69, 053616 (2004).

[11] A. R. Kolovsky, Bloch oscillations in the Mott-insulator regime, Phys. Rev. A 70, 015604 (2004).

[12] C. P. Rubbo, S. R. Manmana, B. M. Peden, M. J. Holland, and A. M. Rey, Resonantly enhanced tunneling and transport of ultracold atoms on tilted optical lattices, Phys. Rev. A 84, 033638 (2011).

[13] M. Kolodrubetz, D. Pekker, B. K. Clark, and K. Sengupta, Nonequilibrium dynamics of bosonic Mott insulators in an electric field, Phys. Rev. B 85, 100505(R) (2012).

[14] F. Meinert, M. J. Mark, E. Kirilov, K. Lauber, P. Weinmann, A. J. Daley, and H.-C. Nägerl, Quantum Quench in an Atomic One-Dimensional Ising Chain, Phys. Rev. Lett. 111, 053003 (2013).

[15] F. Meinert, M. J. Mark, E. Kirilov, K. Lauber, P. Weinmann, M. Gröbner, A. J. Daley, and H.-Ch. Nägerl, Observation of manybody long-range tunneling after a quantum quench, Science 344 , 1259 (2014).
[16] S. Pielawa, T. Kitagawa, E. Berg, and S. Sachdev, Correlated phases of bosons in tilted frustrated lattices, Phys. Rev. B 83, 205135 (2011).

[17] M. Atala, M. Aidelsburger, M. Lohse, J. T. Barreiro, B. Paredes and I. Bloch, Observation of chiral currents with ultracold atoms in bosonic ladders, Nat. Phys. 10, 588 (2014).

[18] D. N. Maksimov, E. N. Bulgakov, and A. R. Kolovsky, WannierStark states in double-periodic lattices. II: Two-dimensional lattices, Phys. Rev. A 91, 053632 (2015).

[19] If one considers the whole Hilbert space, higher occupations $n>$ 2 of the lattice sites become possible. This process, however, is suppressed by including into the Bose-Hubbard Hamiltonian an additional term $U^{\prime} \sum \hat{n}_{l, m}\left(\hat{n}_{l, m}-1\right)\left(\hat{n}_{l, m}-2\right)$, which takes into account corrections to the interactions energy of triply occupied sites [16]. The relation between the interaction constants $U$ and $U^{\prime}$ has been experimentally studied in the recent work [20].

[20] M. J. Mark, E. Haller, K. Lauber, J. G. Danzl, A. Janisch, H. P. Büchler, A. J. Daley, and H.-C. Nägerl, Preparation and Spectroscopy of a Metastable Mott-Insulator State with Attractive Interactions, Phys. Rev. Lett. 108, 215302 (2012). 